\title{
Non-cardiac comorbidities in adults with inherited and congenital heart disease: report from a single center experience of more than 800 consecutive patients
}

\author{
Rhoia Clara Neidenbach ${ }^{1 \#}$, Eckart Lummert ${ }^{1 \#}$, Matthias Vigl' ${ }^{2}$, Reinhard Zachoval ${ }^{3}$, Michael Fischereder $^{4}$, \\ Andrea Engelhardt ${ }^{1}$, Claudia Pujol ${ }^{1}$, Renate Oberhoffer ${ }^{1,5}$, Nicole Nagdyman ${ }^{1}$, Peter Ewert ${ }^{1}$, Michael \\ Hauser $^{1}$, Harald Kaemmerer ${ }^{1}$ \\ ${ }^{1}$ Department of Pediatric Cardiology and Congenital Heart Disease, German Heart Center Munich, Technical University Munich, Munich, Germany; \\ ${ }^{2}$ Deutsches Institut für Ernährungsforschung (DIFE), Potsdam-Rehbrücke, Nuthetal, Germany; ${ }^{3}$ Department of Gastroenterology, ${ }^{4}$ Department of \\ Nephrology, Ludwig-Maximilians-University Munich, Munich, Germany; Preventive Pediatrics, Technical University Munich, Munich, Germany \\ Contributions: (I) Conception and design: RC Neidenbach, H Kaemmerer; (II) Administrative support: M Vigl, A Engelhardt, C Pujol; (III) \\ Provision of study materials or patients: RC Neidenbach, H Kaemmerer, E Lummert, A Engelhardt, C Pujol, N Nagdyman, P Ewert, M Hauser; \\ (IV) Collection and assembly of data: RC Neidenbach, H Kaemmerer, E Lummert, C Pujol, N Nagdyman, M Hauser; (V) Data analysis and \\ interpretation: RC Neidenbach, H Kaemmerer, E Lummert, M Vigl, R Zachoval, M Fischereder, R Oberhoffer, N Nagdyman, P Ewert, M Hauser; \\ (VI) Manuscript writing: All authors; (VII) Final approval of manuscript: All authors.
}

\#These authors contributed equally to this work.

Correspondence to: Rhoia Clara Neidenbach, PhD. Department of Pediatric Cardiology and Congenital Heart Disease, German Heart Centre Munich, Munich, Germany. Email: neidenbach@dhm.mhn.de.

Background: As a result of improved surgical and therapeutical management, more than $90 \%$ of patients with congenital heart disease (CHD) reach adulthood. However, the natural course of CHD is complicated by noncardiac medical problems. Aim of the study was to evaluate noncardiac comorbidities in a contemporary cohort of adults with CHD (ACHD).

Methods: In a tertiary care center for ACHD, 821 consecutive patients, admitted to the outpatient clinic, were evaluated for clinically relevant noncardiac comorbidities.

Results: The consecutively included patients (age: range, 15-80 years; 56\% female) represent all types and severity grades of acyanotic and cyanotic CHD. A considerable proportion of ACHD had significant noncardiac comorbidities, which have the potential to profoundly influence the natural course of the underlying disease. In $95.5 \%$, relevant non-cardiac comorbidities were apparent, that could be related to 16 special medical fields as endocrinologic/metabolic disease, gastroenterology/hepatology, gynecology/ obstetrics, angiology, orthopedics, neurology/psychiatry and others. Most frequently seen comorbidities were endocrine and metabolic disorders (43.97\%).

Conclusions: Non-cardiac comorbidities are increasingly common in ACHD. The data revealed non-cardiac comorbidities as they were presented in the cohort of ACHD seen in a tertiary center. The study proves that ACHD with significant non-cardiac comorbidities need multidisciplinary care by medical organ specialists, aside the congenital cardiologist, with a deep knowledge about congenital heart defects, the special effects of the organ disease on the particular heart defect and, how the heart defect may affect the course of the particular organ disease. The study may create the basis for the development of screening programs for comorbidities in ACHD as well as a multidisciplinary concept for diagnosis and treatment of concomitant disorders or for disease prevention. Particularly disease prevention may improve quality of life as well as the further fate of the affected patients.

Keywords: Congenital heart disease (CHD); adults; non-cardiac comorbidity; health care system; prevention

Submitted Feb 06, 2018. Accepted for publication Mar 16, 2018.

doi: $10.21037 / \mathrm{cdt} .2018 .03 .11$

View this article at: http://dx.doi.org/10.21037/cdt.2018.03.11 


\section{Introduction}

Very recently a scientific statement from the American Heart Association about noncardiac complications in adults with congenital heart disease (ACHD) disease has been published (1). However, although the problem has been well recognized and described, no quantitative data are provided.

Congenital heart diseases (CHDs) are the most frequent inborn malformations, diagnosed in the newborn period. There are about 1.9 million children and 2.3 million ACHD registered in Europe, with a rapidly cumulating number of ACHD (2). As a result of improved surgical and therapeutical management for congenital heart lesions, more than $90 \%$ of patients achieve adulthood (3).

Surgical reparative techniques for congenital heart lesions were introduced several decades ago and have subsequently undergone continuous modification. Nevertheless, most patients with CHD are not cured and suffer from residual morphologic or hemodynamic sequelae (4).

While most of the problems can be related directly to the specific CHD, non-cardiac comorbidities may become increasingly crucial with ongoing age (5). Nevertheless, regarding this aspect, only few contemporary data are available (6).

The objective of the study was to describe the occurrence of non-cardiac comorbidities in ACHD, in order to provide a basis for adequate preventive medical measures. Since non-cardiac comorbidities may become increasingly crucial with ongoing age, we assessed the prevalence and spectrum of non-cardiac co-morbidities in ACHD.

\section{Methods}

In this cross-sectional clinical study, 821 consecutive patients from a tertiary care center for ACHD (Department of Pediatric Cardiology and Congenital Heart Disease, German Heart Center Munich, Technical University Munich, Munich, Germany) were included. The study was approved by the ethical board of the Technical University of Munich. All patients received an information letter about the aim of the study and gave written informed consents. Requirement for inclusion were the existence of a $\mathrm{CHD}$, and adult age ( $>18$ years) or an appropriate physical maturity.

The medical records were reviewed for patient demographics, cardiac and non-cardiac comorbidities, surgical, interventional or electrophysiological procedures, clinical condition and medication. As "relevant", all comorbidities and diagnoses were classified, which had been included in the medical reports as they were classified by an experienced cardiologist to be important enough for future prophylactic or therapeutic patient management.

Patients were assigned to 1 out of 4 functional classes (FCs), according to the recommendations of Perloff et al. (7). For further analysis, patients were classified according to their cardiac diagnosis following the recommendations of the American College of Cardiology (ACC) (8).

For statistical analysis, all descriptive data were expressed in mean-values and standard deviations. Discrete variables are expressed as absolute and relative frequencies. For the statistical analysis SPSS ${ }^{\circledR}$ Version 21 (SPSS Inc., Chicago, IL, USA) was used.

\section{Results}

\section{Patient characteristics}

A total of 821 patients (56.4\% female) were included into the study. The median age was 34.8 years (mean $37.3 \pm 11.6$ years; range, $15.5-80$ years). Two of the included patients were younger than 18 years, but had been judged before by their caring pediatric cardiologist to be physically and psychologically so mature that they had been transferred to the adult department already.

CHDs were subclassified into six main groups, including (I) complex forms of CHD ( $\mathrm{n}=168)$; (II) left ventricular outflow tract obstruction ( $\mathrm{n}=159)$; (III) right ventricular outflow tract obstruction ( $\mathrm{n}=158)$; (IV) pre-tricuspid shunts ( $\mathrm{n}=92)$; (V) post-tricuspid shunts $(\mathrm{n}=92)$; and (VI) miscellaneous CHD ( $\mathrm{n}=152)$.

In the entire cohort, 752 (91.6\%) patients were acyanotic, whereas $69(8.4 \%)$ were cyanotic with a percutaneous oxygen saturation of below $92 \%$.

A syndromal disorder with cardiovascular involvement was present in 94 out of 821 patients [Marfan syndrome (8.2\%), Down syndrome (2.7\%), Ehlers-Danlos syndrome $(0.24 \%)$, Noonan syndrome $(0.12 \%)$, Turner syndrome $(0.12 \%)$, and Williams-Beuren syndrome $(0.12 \%)]$.

No operation/intervention up to the time of the study had 180 (21.9\%) patients with CHD, while 579 $(70.5 \%)$ had corrective or palliative surgery. For the treatment of their CHD, 265 (45.8\%) patients needed only one operation, while in 310 patients up to six surgical procedures were performed. In 259 (31.5\%) of the patients with CHD an interventional procedure was performed; 156 (19\%) had only one intervention, in $103(12.5 \%)$ up to six interventions. 
Table 1 Clinical classification

\begin{tabular}{lc}
\hline Variables & Value \\
\hline Age, years & $16-80$ \\
Gender ( $\mathrm{n}=821), \mathrm{n}(\%)$ & $463(56.4)$ \\
Female & $358(43.6)$ \\
Male & \\
Cyanosis ( $\mathrm{n}=821), \mathrm{n}(\%)$ & $752(91.6)$ \\
Acyanotic & $69(8.4)$ \\
Cyanotic & \\
Functional class (Perloff) ( $\mathrm{n}=821), \mathrm{n}(\%)$ & $730(88.9)$ \\
I/II & $91(11.1)$ \\
III & 0 \\
IV & \\
Severity code of CHD according & \\
ACC classification ( $\mathrm{n}=720), \mathrm{n}(\%)$ & $137(19.0)$ \\
Simple & $381(52.9)$ \\
Intermediate & $202(28.1)$ \\
Severe & \\
Severity code of miscellaneous CHD ( $\mathrm{n}=101), \mathrm{n}(\%)$ \\
Simple \\
Severe
\end{tabular}

CHD, congenital heart disease; ACC, American College of Cardiology.

\section{Functional patient status}

According to Perloff's classification, 730 (88.9\%) patients were in FC I/II, 91 (11.1\%) in FC III and none in FC IV (Table 1). In addition, $720(87.7 \%)$ out of the 821 could be classified according to the ACC severity code: $137 / 720$ were classified as simple (19.0\%), 381/720 as intermediate $(52.9 \%)$ and $202 / 720$ as severe $(28.1 \%)$.

The remaining 101 ACHD could not be classified according the ACC severity code, but were graded according the Munich classification system in simple $(30 / 101,29.7 \%)$ and severe $(71 / 101,70.3 \%)$ forms of CHD (Table 1).

\section{Non-cardiac comorbidities}

In 782 (95.2\%) out of 821 patients, significant non-cardiac comorbidities were apparent, which could be assigned to 16 special medical fields. Many patients suffered from more than one non-cardiac comorbidity (Table 2).

In the present study, as many as 361/821 (44\%) patients had metabolic (e.g., diabetes mellitus, hyperlipidemia, hyperuricemia) and/or endocrine disorders (particularly hypo- and hyperthyroidism). In 344/821 of the patients $(42 \%)$ one or more endocrine or metabolic disorders was present. In 170/821 (21\%) thyroid disorders could be verified. A high incidence of thyroid dysfunction was found in patients treated with Amiodarone $(10 / 28,36 \%)$ and in patients with Down syndrome (19/22, 86\%). Metabolic disorders (hyperlipidemia, hyperuricemia) were present in $151 / 821$ (18\%), diabetes mellitus in only $23 / 821$ (2.8\%). Gastroenterologic and hepatologic comorbidities were quite common (253/821,31\%). Gynecologic and obstetrical issues were seen in 245/463 (53\%) of all female patients. One hundred and thirty-five out of 821 patients $(16 \%)$ had arterial hypertension. Peripheral venous (78/821, 9.5\%), or arterial $(24 / 821,2.9 \%)$ vascular diseases were present in $12.4 \%$ (102/821). Musculoskeletal problems, particularly deformities of the spine or sequels of previous sternotomy or thoracotomy were found in $187 / 821$ (22.8\%) of the subjects. Neurological $(148 / 821,18 \%)$ and psychiatric problems $(52 / 821,6.3 \%)$ were seen very often $(200 / 821,24 \%)$, while relevant pleuro-pulmonic disease $(126 / 831,15 \%)$, and renal disorders (55/821, 6.7\%) were comparatively rare.

Oncological diseases, including tumors of the colon, testicles, prostat, cervix or thyroid, were exceptional and could be verified in 38/821 (4.6\%) of the included patients.

\section{Discussion}

\section{Resida, sequels and comorbidities in adult CHD}

Despite all progresses in surgical, interventional and pharmacological management, many patients with CHD are not cured and have significant resida and sequels from the underlying anomaly, a reduced quality of life and life expectancy, and the hospitalization rate due to complications of ACHD is high.

\section{Non-cardiac comorbidities in adult CHD}

Largely underestimated in this regard is the importance of non-cardiac comorbidities, defined as the presence of additional disorders co-occurring with the CHD, as they may induce or aggravate deterioration in the course of the underlying CHD. Therefore, also in patients with CHD early detection and adequate management of comorbidities is of outstanding importance in the follow-up (9). 
Table 2 Non-cardiac comorbidities

\begin{tabular}{|c|c|}
\hline Speciality & $n$ \\
\hline Endocrinology/metabolic disease & 344 \\
\hline Thyroid gland & 170 \\
\hline Metabolic disease & 151 \\
\hline Diabetes mellitus & 23 \\
\hline Gastroenterology/hepatology & 253 \\
\hline Gastro-intestinal tract & 99 \\
\hline Hepatology & 99 \\
\hline Biliary tract & 51 \\
\hline Pancreas & 4 \\
\hline Gynecology & 49 \\
\hline Obstetrics & 183 \\
\hline Breast & 13 \\
\hline Angiology & 237 \\
\hline Arterial hypertension & 135 \\
\hline Arteries & 24 \\
\hline Veins & 78 \\
\hline $\begin{array}{l}\text { Muscular-skeletal disease, orthopedics, } \\
\text { traumatology }\end{array}$ & 233 \\
\hline Muscular-skeletal/orthopedic & 187 \\
\hline Traumatology & 46 \\
\hline Infectiology & 121 \\
\hline Allergology & 85 \\
\hline Neurology & 148 \\
\hline Psychiatry & 52 \\
\hline Otorhinolaryngology & 96 \\
\hline Ophthalmology & 53 \\
\hline Hematology & 116 \\
\hline Erythrocytes & 40 \\
\hline Coagulation/thrombocytes & 40 \\
\hline Iron deficiency & 36 \\
\hline Pulmonary/pleural disease & 126 \\
\hline Lung & 112 \\
\hline Pleura & 6 \\
\hline Diaphragm & 8 \\
\hline
\end{tabular}

Table 2 (continued)
Table 2 (continued)

\begin{tabular}{lc}
\hline Speciality & $\mathrm{n}$ \\
\hline Urology/nephrology & 79 \\
Kidney & 55 \\
Urogenital & 24 \\
Dermatology & 53 \\
Oncology & 38 \\
Malignant tumor (colon, testicle, prostate & 9 \\
gland, cervix, thyroid gland) & 29 \\
Miscellaneous & 7 \\
Orthodontic disease & 6 \\
Rheumatology & 131 \\
Miscellaneous & \\
\hline
\end{tabular}

Moreover, recognition of relevant comorbidities at an early stage, should be a main aim of future efforts in order to timely take preventive measures to reduce the morbidity and mortality of CHD in the long term.

Until to date, only few studies have highlighted the importance and clinical impact of non-cardiac comorbidities in patients with CHD. One study, including patients with CHD older than 65 years of age, indicated, that the impact of non-cardiac comorbidities on mortality was even more relevant than the underlying cardiac disease during a follow-up period of 15 years (10). Another study, including 201 patients urgently demonstrated admitted to hospital, demonstrated in $63 \%(\mathrm{n}=126)$ noncardiac comorbidities as reasons for admission $(11,12)$. Their management required a tight cooperation with other specialized departments, such as neurology, ophthalmology, otorhinolaryngology, gynecology, psychiatry, dermatology and orthopedics. These study outcomes were confirmed by an Asian study published in 2011 (13).

In the current study, out of 821 patients with CHD, $782(95.2 \%)$ had significant non-cardiac comorbidities. Within the cohort, 641 (78\%) had previous surgical or interventional treatment of their CHD, $180(22 \%)$ had a native CHD, and 94 (11\%) had a syndrome with cardiovascular involvement, of which 67 (8.2\%) subjects had a Marfan syndrome. The apparent non-cardiac comorbidities were related to 16 different medical subspecialities. Endocrinologic and metabolic disorders $(n=361)$ were present the most, followed by gastroenterologic 
and hepatologic $(n=253)$, gynecologic and obstetric $(n=245)$, musculoskeletal and orthopedic $(n=233)$, infectiologic, immunologic and allergologic $(\mathrm{n}=206)$, neuro-psychiatric $(n=200)$, othorhinopharyngeal and ophthalmologic $(n=149)$, hematologic $(\mathrm{n}=138)$, pleuro-pulmonic $(\mathrm{n}=126)$, angiologic $(\mathrm{n}=102)$, uro-nephrologic $(\mathrm{n}=79)$, dermatologic $(\mathrm{n}=53)$ and oncologic disease $(n=38)$. One hundred and forty-four had more than one non-cardiac comorbidity (Table 2). The findings of the current study differ from published data were lung, kidney, liver, as well as neurological and angiological disorders were seen the most (14).

\section{Metabolic and endocrinologic disorders}

The number of patients with metabolic and endocrinologic disorders in the present study was unexpectedly high in comparison to literature, where the combination with CHD and metabolic (e.g., diabetes mellitus, hyperlipidemia, hyperuricemia) and endocrinologic disorders (in particular thyroid hypo- and hyperfunction) are estimated as marginal. In contrast, $44 \%(361 / 821)$ of our patients suffered of one or more endocrine or metabolic disorders, including thyroid-dysfunction, hyperlipidemia, hyperuricemia and diabetes mellitus. In the present study, prevalence of diabetes mellitus in CHD $(2.8 \%, 23 / 821)$ was equivalent to the findings of Billett $e t$ al., who described a significantly higher incidence, if compared to the general population (15).

The high frequency of hyperuricemia in our study population is presumably not indicative of genuine gout, but results at least as a consequence of renal dysfunction in cyanotic patients.

The high incidence of thyroid disorders in patients with CHD, particularly in patients with Down syndrome $(86 \%$, $19 / 22$ ), reflects the fact, that hypothyroidism is the most frequent combined with endocrine disorder, affecting only $0.25-1.0 \%$ of the otherwise healthy population, and is also related to the frequent use of Amiodarone $(36 \%, 10 / 28)$ in CHD.

\section{Pulmonary disorders}

According to literature, there is a high incidence of pulmonary disorders in ACHD, particularly restrictive lung disease. Patients with Fontan circulation (89\%), Tetralogy of Fallot (76\%), pulmonary stenosis $(60 \%)$ or coarctation repair are mainly affected (16). The exact incidence remains unclear (17). The etiology of pleuropulmonary disease is multifactorial and results from surgical alterations of the lung, the diaphragm or pleura, and from neuromuscular disorders of respiratory muscles, or spinal and skeletal deformities after sternotomy (18). The individual hemodynamic of a CHD may lead to a developmental or functional lung disorders. In Tetralogy of Fallot or pulmonary atresia, reduced lung perfusion can result in hypoplastic pulmonary arteries, while in patients with significant recirculation, pulmonary vascular disease may develop. Pulmonary arterial hypertension (PAH), respectively pulmonary vascular disease, was seen in about $10 \%$ of adult patients with CHD and leads to a negative effect on quality of life and survival, especially in Eisenmenger syndrome (17). Pharmacotherapy (e.g., Amiodarone) may additionally influence the lung function negatively (19). Those pulmonary disorders have a negative impact on morbidity and mortality in CHD patients, and a moderate to severe impairment of the lung function is an independent causal variable for survival of ACHD (20).

\section{Renal disorders}

Renal disorders are common in ACHD. Billett et al. could provide evidence of a significantly increased prevalence of renal disease compared to healthy controls (15). In comparison to the general population, patients with noncyanotic CHD experience renal disorders 18 times, and patients with cyanotic CHD 35 times more often (21). Myocardial dysfunction of the systemic ventricle with reduced cardiac output and reduced renal perfusion, activation of the renin-angiotensin system with volume overload, interference of the sodium balance, arterial hypertension, anemia and elevated systemic-venous pressure (e.g., in Fontan circulation) are causing factors $(14,21)$. In cyanotic CHD, chronic hypoxia and reactive erythrocytosis with elevated blood-viscosity induce renal disorders by an impairment of renal perfusion and filtration $(13,21)$. Mortality is significantly increased in patients with CHD and impaired renal function, especially in those with complex CHD, Eisenmenger syndrome, Fontan-circulation and valvular diseases (21).

The surprising low number of patients with renal disease in the present cohort may be explained by the fact, that only patients, who needed a targeted therapy or nephrology care were listed as relevant.

\section{Liver and biliary tract disease}

In concordance with current literature, liver and biliary tract disease occurring in many patients included in the 
present study (22). Liver-congestion, fibrosis and cirrhosis are frequent in patients with univentricular heart after Fontan operation, after atrial repair (Senning or Mustard operation) in complete transposition of the great arteries (TGA), as well as in severe pulmonary hypertension and Eisenmenger syndrome (23).

Disorders of the biliary tract are underestimated in CHD. Particularly cyanotic patients are prone to develop gall stones, because of the increased erythrocyte turnover and hyperbilirubinemia. High concentrations of unconjugated bilirubin in the bile increases the risk for gall stones and cholecystitis, which may induce life-threatening complications in case of septicemia (24).

\section{Neurological and psychiatric disorders}

Neurological problems are important sequelae in ACHD, since the incidence is 10 to 100 times higher, if compared to a healthy population. In a retrospect analysis of more than 23,000 CHD patients, Hoffmann et al. demonstrated an incidence of cerebrovascular accidents in more than $2 \%$. Cyanotic patients, with a high rate of ischemic stroke and consecutive longtime disability, are especially affected (25).

As our study shows, psychiatric disorders (52/821, $6.3 \%$ ) are very common and should seriously be taken into consideration, as they may considerably influence the cognitive and psychosocial development of CHD patients. The prevalence is significantly higher compared to the general population (26). This may result from biological and hemodynamic abnormalities during childhood, as well as the necessity of frequent health care, hospitalization, pharmacological and invasive medical therapy (26). Additional etiologic factors are cerebral hypoperfusion and embolism, in part induced by cardiopulmonary bypass and cardiac arrest in accidental hypothermia (27).

From acquired heart diseases, it is well known, that depression has a negative influence on the cardiovascular function, morbidity and mortality. In depressive patients with heart failure, the neglection of medical advice in terms of medication and life-style, has significantly increased the risk of a recurrent cardiac event and a higher mortality (28).

\section{Peripheral venous or arterial vascular disease}

In our study, 12.4\% (102/821) of patients had a peripheral venous, or arterial vascular disease. This has not yet been described in literature. Jeopardized are patients, due to multiple vascular manipulations, as cardiac catheterization or cannulation for cardiac surgery. Patients after Fontan operation, after atrial switch with inferior baffle obstruction, or in Ebstein's anomaly with tricuspid valve dysfunction are at risk of venous congestion (29).

\section{Musculoskeletal or orthopedic disorders}

Musculoskeletal or orthopedic problems were found in $22.8 \%(187 / 821)$ of ACHD. Particularly common were deformities of the spine after sternotomy or thoracotomy. Herrera-Soto et al. found scoliosis, kyphosis or a combination of both in $34 \%$ of patients after thoracic surgery; prevalence was 10 times higher than in idiopathic scoliosis (30). Skeletal anomalies are very frequent, especially in Marfan syndrome. In our cohort, $52 \%$ (35/67) of patients with Marfan syndrome had orthopedic problems.

\section{Hematologic problems and oncological disease}

Hematologic problems are often seen in cyanotic patients and in Eisenmenger syndrome. These patients develop reactive erythrocytosis, iron deficiency or folic acid deficiency (24), often induced by a high red cell turnover in reactive erythrocytosis.

Oncological diseases are rare in patients with CHD and could be verified in our study in only $4.6 \%$ (38/821), including tumors of the colon, testicles, prostate, cervix or thyroid. Also in literature, only few data exist in this regard, but a rising frequency with increasing age of patients has to be expected (31).

\section{Gynecologic and obstetric problems}

As anticipated, many women with CHD develop gynecologic and obstetric problems, in the current study represented in $53 \%(245 / 463)$ of all females. Within the general population, $0.2-4 \%$ of all pregnancies develop cardiovascular problems. In pregnant women with CHD the frequency is $11-20 \%$ (32), depending on the type of CHD, the functional status of the mother and the results of previous surgical or interventional procedures.

\section{Health care relevance of the study}

The study proves that ACHD have significant non-cardiac comorbidities and need multidisciplinary care. A scientific statement from the American Heart Association about diagnosis and management of noncardiac complications in 
ACHD underlines the importance of the subject (1).

Recommendations for an integrated interdisciplinary care of ACHD do exist, e.g., in Canada, Central Europe, and in the US, also underlining the importance of noncardiac comorbidities. Nevertheless, in many countries, ACHD are still undersupplied within the present health care system. A recent study focusing on primary health care ACHD in Germany demonstrated, that despite all efforts of an integrated patient-centered care, the percentage of ACHD, treated in a specific certified center is minor (33).

Therefore, it is of significant importance, to integrate ACHD into specialized care, where specific noncardiac comorbidities can be identified and managed. A comprehensive network must be developed for this growing population, with extensive collaboration between experienced cardiologists and specialists from other medical fields, with the ability and experience to accomplish noncardiac problems.

For this purpose, also the medical organ specialists aside the congenital cardiologist needs a deep knowledge about congenital heart defects. He has to know in detail the special effects of the organ disease on the particular heart defect and also, how the heart defect may affect the course of the particular organ disease. Depending on the severity of complications, within an ACHD-center a strong collaboration is indispensable in the field of pulmonology, nephrology, hepatology and neurology. This is all the more important the more complex the congenital cardiac disorder is.

The experience at the German Heart Center Munich, where a network structure across medical disciplines was built over many years for the supply of ACHD, indicates that this is a hard to reach goal.

Finally, general practitioners, who take care of ACHD patients in a great extent, should be aware of the importance of non-cardiac comorbidities. Primary and secondary prevention in ACHD will become increasingly important in order to reduce the burden of disease as well as the socioeconomic burden and costs.

\section{Study limitations}

This study has limitations as it is retrospective, uncontrolled and not population based.

As the co-morbidities were taken from the medical records, some comorbidity may have been classified incorrectly or even missed. The sample of patients may not represent the pattern of ACHD in the community and does not represent the typical population of CHD seen by a general practitioner or by a normal cardiologist.

As the current study focused on adults under follow-up of a tertiary centre for ACHD, the more complex anomalies reported here are likely rare in community-based hospitals or even in departments for cardiology.

A generalization of the conclusions and their transfer to adult patients with CHD living outside of Germany or in different culture groups may be questionable.

\section{Conclusions}

The current study presents "real-life data", defined as recorded data from the available electronic health records and medical chart reviews relating to the health status and/or the delivery of health care that patients received, based on a large sample size of ACHD, highlighting the importance of non-cardiac comorbidities as seen in a tertiary center for CHD. Co-morbidities are very often the sequelae of underlying congenital cardiac disease or result as a consequence of previous surgical or interventional procedures. It is obvious, that chronic heart disease influence other organ systems and vice versa, and has a significant impact on quality of life, morbidity and mortality of the patients with CHD. The study may be of importance to develop screening programs for co-morbidities and to create a multidisciplinary concept for diagnosis and treatment. Moreover, the present study provides the basis for further improvement of medical care and also for a further development of primary and secondary preventive measures.

\section{Acknowledgements}

Funding: The authors would like to thank the "Deutsche Herzstiftung" as well as the patient organization "Herzkind e.V." for research funding (No. F-30-15) in the field of ACHD.

\section{Footnote}

Conflicts of Interest: The authors have no conflicts of interest to declare.

Ethical Statement: The study was approved by the Ethical Committee of the Faculty of Medicine of the Technical University of Munich on the 15th of November 2010 (No. 2977/10). All patients received an information letter about the aim of the study and gave written informed consents. 


\section{References}

1. Lui GK, Saidi A, Bhatt AB, et al. Diagnosis and Management of Noncardiac Complications in Adults With Congenital Heart Disease: A Scientific Statement From the American Heart Association. Circulation 2017;136:e348-92.

2. Baumgartner H. An important attempt to improve the outcome of congenital heart disease in Europe. Eur Heart J 2014;35:674-5.

3. Schmaltz AA, Bauer UM. Adults with congenital heart disease: treatment and medical problems. Herz 2013;38:639-51; quiz 652-4.

4. Perloff JK, Warnes CA. Challenges posed by adults with repaired congenital heart disease. Circulation 2001;103:2637-43.

5. Kaemmerer H, Hess J. Congenital heart disease. Transition from adolescence to adulthood. Internist (Berl) 2009;50:1221-2, 1224-7.

6. Lummert E, Hauser M, Vigl M, et al. Noncardiac Comorbidities of Congenital Heart Disease in Adults. Am J Cardiol 2014;113:S109.

7. Perloff J, Child J, Aboulhson J. Congenital heart disease in adults. 3rd edition. Philadelphia: WB Saunders, 2009.

8. Warnes CA, Liberthson R, Danielson GK, et al. Task force 1: the changing profile of congenital heart disease in adult life. J Am Coll Cardiol 2001;37:1170-5.

9. Gilboa SM, Salemi JL, Nembhard WN, et al. Mortality resulting from congenital heart disease among children and adults in the United States, 1999 to 2006. Circulation 2010;122:2254-63.

10. Afilalo J, Therrien J, Pilote L, et al. Geriatric congenital heart disease: burden of disease and predictors of mortality. J Am Coll Cardiol 2011;58:1509-15.

11. Kaemmerer H, Bauer U, Pensl U, et al. Management of emergencies in adults with congenital cardiac disease. Am J Cardiol 2008;101:521-5.

12. Verheugt CL, Uiterwaal CS, van der Velde ET, et al. The emerging burden of hospital admissions of adults with congenital heart disease. Heart 2010;96:872-8.

13. Koh AS, Yap BT, Le Tan J. Emergency admissions in Asians with adult congenital heart disease. Int J Cardiol 2011;151:54-7.

14. Cohen SB, Ginde S, Bartz PJ, et al. Extracardiac complications in adults with congenital heart disease. Congenit Heart Dis 2013;8:370-80.

15. Billett J, Cowie MR, Gatzoulis MA, et al. Comorbidity, healthcare utilisation and process of care measures in patients with congenital heart disease in the UK: crosssectional, population-based study with case-control analysis. Heart 2008;94:1194-9.

16. Pianosi PT, Johnson JN, Turchetta A, et al. Pulmonary function and ventilatory limitation to exercise in congenital heart disease. Congenit Heart Dis 2009;4:2-11.

17. Ginde S, Bartz PJ, Hill GD, et al. Restrictive lung disease is an independent predictor of exercise intolerance in the adult with congenital heart disease. Congenit Heart Dis 2013;8:246-54.

18. Kaemmerer H, Gorenflo M, Hoeper M, et al. Pulmonary arterial hypertension in patients with congenital heart disease: current issues and health care situation. Dtsch Med Wochenschr 2013;138:1247-52.

19. Schwaiblmair M, Berghaus T, Haeckel T, et al. Amiodarone-induced pulmonary toxicity: an underrecognized and severe adverse effect? Clin Res Cardiol 2010;99:693-700.

20. Alonso-Gonzalez R, Borgia F, Diller GP, et al. Abnormal lung function in adults with congenital heart disease: prevalence, relation to cardiac anatomy, and association with survival. Circulation 2013;127:882-90.

21. Dimopoulos K, Diller GP, Koltsida E, et al. Prevalence, predictors, and prognostic value of renal dysfunction in adults with congenital heart disease. Circulation 2008;117:2320-8.

22. Gewillig M, Goldberg DJ. Failure of the fontan circulation. Heart Fail Clin 2014;10:105-16.

23. Kaulitz R, Haber P, Sturm E, et al. Serial evaluation of hepatic function profile after Fontan operation. Herz 2014;39:98-104.

24. Kaemmerer H, Niwa K, Hess J. Das EisenmengerSyndrom - vom Symptom zu Diagnose und Therapie. Bremen: UniMed, 2011.

25. Hoffmann A, Chockalingam P, Balint OH, et al. Cerebrovascular accidents in adult patients with congenital heart disease. Heart 2010;96:1223-6.

26. Marino BS, Lipkin PH, Newburger JW, et al. Neurodevelopmental outcomes in children with congenital heart disease: evaluation and management: a scientific statement from the American Heart Association. Circulation 2012;126:1143-72.

27. Shillingford AJ, Wernovsky G. Academic performance and behavioral difficulties after neonatal and infant heart surgery. Pediatr Clin North Am 2004;51:1625-39, ix.

28. Lane DA, Chong AY, Lip GY. Psychological interventions for depression in heart failure. Cochrane Database Syst Rev 2005;(1):CD003329. 
29. Valente AM, Bhatt AB, Cook S, et al. The CALF (Congenital Heart Disease in Adults Lower Extremity Systemic Venous Health in Fontan Patients) study. J Am Coll Cardiol 2010;56:144-50.

30. Herrera-Soto JA, Vander Have KL, Barry-Lane P, et al. Retrospective study on the development of spinal deformities following sternotomy for congenital heart disease. Spine (Phila Pa 1976) 2007;32:1998-2004.

31. Lee YS, Chen YT, Jeng MJ, et al. The risk of cancer in patients with congenital heart disease: a nationwide

Cite this article as: Neidenbach RC, Lummert E, Vigl M, Zachoval R, Fischereder M, Engelhardt A, Pujol C, Oberhoffer R, Nagdyman N, Ewert P, Hauser M, Kaemmerer H. Noncardiac comorbidities in adults with inherited and congenital heart disease: report from a single center experience of more than 800 consecutive patients. Cardiovasc Diagn Ther 2018;8(4):423-431. doi: 10.21037/cdt.2018.03.11 population-based cohort study in Taiwan. PLoS One 2015;10:e0116844.

32. Kaemmerer H, Bauer U, Stein JI, et al. Pregnancy in congenital cardiac disease: an increasing challenge for cardiologists and obstetricians -- a prospective multicenter study. Z Kardiol 2003;92:16-23.

33. Neidenbach R, Kaemmerer H, Pieper L, et al. Striking Supply Gap in Adults with Congenital Heart Disease? Dtsch Med Wochenschr 2017;142:301-3. 